\title{
Modelling of Complex Systems Given as a Mealy Machine with Linear Decision Diagrams
}

\author{
P. Dziurzanski \\ Faculty of Computer Science and Information Systems, Technical University of Szczecin, \\ Zolnierska 49, 71-210 Szczecin, POLAND \\ pdziurzanski@wi.ps.pl
}

\begin{abstract}
We propose a novel approach to transform an arbitrary Mealy machine, which is a kind of Finite State Machine (FSM) with outputs, into so-called Linear Binary Moment Diagrams (LBMDs). We stress the attractive features of this approach, and demonstrate the results of experiments on benchmarks in comparison with a state-of-the art Reduced Ordered Binary Decision Diagrams technique.
\end{abstract}

\section{Introduction}

Many practical problems in a field of sequential system optimization are described by finite state system over a sequence of state transitions. As classical Finite State Machine (FSM) offers only two outputs, namely 'accepted' and 'unaccepted', modifications of FSM with allows multiple output values are often used instead. The most popular FSMs of that type are Mealy and Moore machines. In this paper, we focus on Mealy machine, but it does not restrict applications of the approach, as each Moore Machine can be transformed into Mealy ones and vice-versa [6].

Although classic algorithms construct an explicit representation [3], these techniques are practical only for small-size problems. In order to overcome the problem, symbolic state graph methods were developed, in which the next state behavior is represented with a Boolean function [2]. Then, a Reduced Ordered Binary Decision Diagram (ROBDD) can be constructed [8]. It is beneficial if all the future operations are carried out only on that diagram.

Although successful for many class of switching functions, the above mentioned method has a number of drawbacks. Firstly, the number of nodes in ROBDD depends on variable ordering. Moreover, for a majority of functions the number of nodes remains exponential. As a result, a construction of ROBDD for characteristic functions is often unsuccessful due to the ROBDD size explosion.

In this paper we deal with word-level Binary Moment Diagrams (BMDs), introduced by Bryant et. al. in [1]. Since BMDs also depend on variable ordering in general, we take advantage of the only class of Decision Diagrams which are insensitive to the variable ordering, known as Linear Decision Diagrams (LDDs). Besides, as our experiments proved, for popular benchmark sets we can build diagrams with lower number of nodes than the typical ROBDD. 


\section{Principles}

\subsection{Characteristic Functions of Mealy Machines}

Mealy machine is a 6-tuple $\left(S, I, O, \delta, \lambda, s_{0}\right)$, where $S$ is the set of states, $I$ is the set of input values, $O$ is the set of output values, $\delta: S \times I \rightarrow O$ is the next state function, $\lambda: S \times I \rightarrow S$ is the next state function, and $s_{0} \in S$ is the initial function.

In order to synthesis of FSM, we need to assign binary codes to the states. This process is called state assignment. After assigning, we can build a truth table, which input set consists of primary inputs $x$ and current states $s$, whereas an output set consists of next states $s^{+}$and primary outputs $y$. After minimizing, truth table is often referred as cube table.

As in mentioned above definition of Mealy machine there are two functions, from which one determines the next state and the other output values, it is convenient to build two characteristic functions for describing a behavior of the automata.

For more details about Mealy machines see, for example, [5]6].

The characteristic function $\chi_{\delta}$ which describes the next state of Mealy machine is the function $f: S \times I \times S \rightarrow \mathbf{B}, f(x, i, y)=1$ iff $y=\delta(x, i)$, where $i \in I, x, y \in S$, and $\mathbf{B}=\{0,1\}$.

The characteristic function $\chi_{\lambda}$ which describes outputs of Mealy machine is the function $f: S \times I \rightarrow \mathbf{B}, f(x, i, z)=1$ iff $z=\lambda(x, i)$, where $i \in I, x \in S, z \in O$, and $\mathbf{B}=\{0,1\}$.

Example 1 Let us consider a State Transition Graph presented in Fig. 17a. After assigning stO with 'O' and st 1 with '1', we obtain a cube table presented in Fig. 17 b. A characteristic function $\chi_{\delta}$ has inputs $x_{1}, x_{2}, s_{1}, s_{1}^{+}$, whereas $\chi_{\lambda}$ has inputs $x_{1}, x_{2}, s_{1}$, $y$. Consequently, the characteristic functions in a SOP forms equal

$$
\begin{aligned}
& \chi_{\delta}=x_{1} x_{2} \bar{s}_{1} \bar{s}_{1}^{+} \vee \bar{x}_{2} \bar{s}_{1} \bar{s}_{1}^{+} \vee \bar{x}_{1} x_{2} \bar{s}_{1} s_{1}^{+} \vee \bar{x}_{1} s_{1} s_{1}^{+} \vee x_{1} x_{2} s_{1} s_{1}^{+}, \\
& \chi_{\lambda}=x_{1} x_{2} \bar{s}_{1} \bar{y}+\vee \bar{x}_{2} \bar{s}_{1} \bar{y} \vee \bar{x}_{1} s_{1} y \vee x_{1} x_{2} s_{1} \bar{y}
\end{aligned}
$$

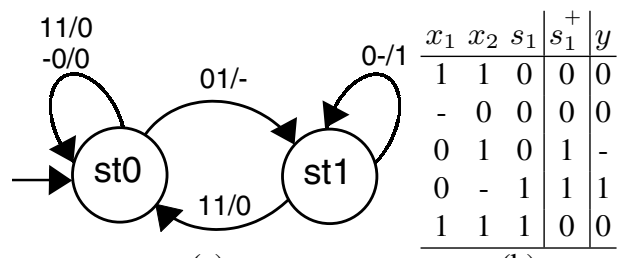

(a)

Fig. 1. An example of a State Transition Graph (a) and its cube table (b)

In our previous work, described in [4], we propose a usage of one characteristic functions, which incorporated both of the next state and outputs ones. Our previous approach makes the process of simulation, i.e. evaluating the outputs and next state performance, much more complex and time-consuming, what makes it inefficient in case of complex systems. 


\subsection{Linear Arithmetic Expressions}

Having a cube table of both of characteristic functions as an input, we can derive a minimal SOP. The SOP consisting of $p$ products is a two-level representation. In the first and the second level $p$ ANDs and one OR functions are implemented, respectively. Each of these functions can be extended to a multi-output function generating a Linear Arithmetic Expressions (LAR) (1), and, therefore, by a Linear Binary Moment Diagram (LBMD).

An arbitrary function can be described by a corresponding arithmetical expression (AR), for example $\bar{x}=1-x, x_{1} \vee x_{2}=x_{1}+x_{2}-x_{1} x_{2}$. In particular cases only these arithmetical expressions have a linear structure, including terms with no more than one variable. LAR for the $n$-variable logic function can be generally described by

$$
d_{0}+d_{1} x_{1}+\ldots+d_{n} x_{n}
$$

where $d_{i}$ are arbitrary integers. Thus the term linearization means a transformation from nonlinear AR into corresponding LAR.

Taking into account that in a case of a word-level expression the value of a given Boolean function are on a specific bit, it is necessary to use a masking operator, which allow us to select an arbitrary bit. The masking operator selecting the $i$-th bit from an $m$-output function $f$ is denoted by $\boldsymbol{\Xi}_{m}^{i}\{f\}$.

The method of describing an $n$-variable elementary function (such as AND and OR) by a LAR is presented in [7]. Now we shortly recall this result.

Theorem 1 (re-formulated from [7]). The n-variable elementary functions can be represented as follows:

(i) $n$-variable AND function $A N D=\bigwedge_{i=1}^{n} x_{i}^{\sigma_{i}}$ by the LAR

$$
L A R_{A N D}=2^{j-1}-n+\sum_{i=1}^{n}\left(\sigma_{i}+(-1)^{\sigma_{i}} x_{i}\right),
$$

generated by an m-output function, in which the function $f$ is in the most left position indicated by the masking operator $\Xi_{m}^{m}\left\{L A R_{A N D}\right\}$;

(ii) $n$-variable OR function $O R=\bigvee_{i=1}^{n} x_{i}^{\sigma_{i}}$ by the $L A R$

$$
L A R_{O R}=2^{j-1}-1+\sum_{i=1}^{n}\left(\sigma_{i}+(-1)^{\sigma_{i}} x_{i}\right)
$$

generated by an m-output function, in which the function $f$ is the most significant bit, that is indicated by the masking operator $\Xi_{m}^{m}\left\{L A R_{O R}\right\}$, where

$$
j=\left\lceil\log _{2} n\right\rceil+1,
$$

and

$$
x_{i}^{\sigma_{i}}= \begin{cases}x_{i} & \text { if } \sigma_{i}=0 \\ \bar{x}_{i} & \text { if } \sigma_{i}=1 .\end{cases}
$$

The proof of the Theorem is given in [7]. 


\subsection{Linear Decision Diagrams}

Our goal is to represent a given sequential digital circuit by a set of two LBMDs. In Decision Diagrams a function is expanded with respect to one splitting variable $x$. As a result of this expansion there are two subfunctions. For a switching function $f, f_{x}$ and $f_{\bar{x}}$ denote the positive and negative cofactor of $f$ with respect to variable $x$, correspondingly. The subfunction $f_{x}$ is obtained by substituting a constant 1 to $x$, whereas $f_{\bar{x}}$ is obtained by substituting a constant 0 to $x$.

In our method, we use masking operators and decision diagrams with arithmetic positive Davio $\left(p D_{A}\right)$ decomposition $f=f_{\bar{x}}+x\left(f_{x}-f_{\bar{x}}\right)$. The method of deriving this equation is presented, for example, in [1].

If arithmetic positive Davio is applied for decomposition of an LAR, it splits the function into two subfunctions $f_{\bar{x}}$ and $\left(f_{x}-f_{\bar{x}}\right)$. It is noticeable, that both of them does not depend on the splitting variable $x$ and the subfunction $\left(f_{x}-f_{\bar{x}}\right)$ is always constant and equal to the coefficient of the variable $x$ in LAR.

\section{Strategy}

Lemma 2 An arbitrary characteristic function can be represented by a double LBMD.

The proof is based on the following two statements:

- Let us have a characteristic function given by a cube table. Each cube in the cube table corresponding to $t$-th, $t \in(1, \ldots, p)$, product $y_{t}=x_{1}^{\alpha_{1}} \wedge \ldots \wedge x_{l}^{\alpha_{l}}, x_{i}^{\alpha_{i}}=\bar{x}_{i}$ when $\alpha_{i}=0$ and $x_{i}^{\alpha_{i}}=x_{i}$ when $\alpha_{1}=1, l \in(1, \ldots, n)$, can be represented by an $l$-node LBMD $A^{X}$, and $p$ products $y_{1}, \ldots, y_{p}$ with no more than $l$ literals each, can be represented by the same LBMD,

- Any set of cubes in the cube table can be treated as a SOP $f=y_{1} \vee \ldots \vee y_{p}$ of $p$ product terms can be represented by an LBMD $B^{Y}$, and an $r$-output function with no more than $p$ products each can be represented by the same LBMD.

Let us consider a characteristic functions. Their outputs describe a SOP with $p$ products with no more than $n$ literals. We extend each product $y_{t}$ (AND function) to an $n_{t}$-output function in order to generate $L A R\left(y_{t}\right)$. The LARs for other products $y_{t}$ form the weighed arithmetic sum:

$$
A^{X}=2^{J_{1}} \operatorname{LAR}\left(y_{1}\right)+\ldots+2^{J_{p}} \operatorname{LAR}\left(y_{p}\right),
$$

and, substituting the $L A R\left(y_{t}\right)$ (1) into the above equation yields the LAR

$$
A^{X}=T W_{0}+T W_{1} x_{1}+\ldots+T W_{n} x_{n},
$$

where the coefficients $T W_{i}, T W_{0}$ are formed as below

$$
\begin{aligned}
& T W_{i}=a_{i, 1} 2^{J_{1}}+\ldots+a_{i, p} 2^{J_{p}}, \\
& T W_{0}=a_{0,1} 2^{J_{1}}+\ldots+a_{0, p} 2^{J_{p}},
\end{aligned}
$$

for $a_{i, t} \in\{0,+1,-1\}$ and positive integer numbers $a_{0, t}$;

$$
\begin{aligned}
& J_{1}=j_{0}=0, \\
& J_{t}=j_{0}+\ldots+j_{t-1},
\end{aligned}
$$

and (equation (4)) 


$$
j_{t}=\left\lceil\log _{2} n_{t}\right\rceil+1
$$

$n_{t}$ is the number of outputs in the function obtained by extending of $y_{t}$. So, AND functions of the first level of the SOP are described by the LAR (7), and, so, by an LBMD $A^{X}$.

Then, characteristic functions $\chi_{\delta}$ and $\chi_{\lambda}$ form a SOP $f=y_{1} \vee \ldots \vee y_{p}$, i.e. a $p$ input OR function. The output $f$ can be extended to a multi-output function, in order to generate $L A R(f)$. They are combined to the LAR:

$$
B^{Y}=L A R(f),
$$

that is equal to

$$
B^{Y}=B W_{0}+B W_{1} y_{1}+\ldots+B W_{p} y_{p}
$$

where the coefficients $J B_{j}$ are formed using equations (9) and

$$
\begin{aligned}
& B W_{t}=b_{t, 1} 2^{J B_{1}}+\ldots+b_{t, r} 2^{J B_{r}} \\
& B W_{0}=b_{0,1} 2^{J B_{1}}+\ldots+b_{0, p} 2^{J B_{r}} .
\end{aligned}
$$

So, all OR functions (the second level of the SOPs) are described by (10), and so we can generate the LBMD $B^{Y}$.

Example 2 (Continuation of Example 1 ) Since both of the characteristic functions $\chi_{\delta}$ and $\chi_{\lambda}$ of the function $f$ include 9 products, there is a need to extend these products $y_{t}$ to an $n_{t}$-output function in order to generate $L A R\left(y_{t}\right)$, where $t=1, \ldots, 9$.

As the $t=1$ st product $y_{1}=x_{1} x_{2} \bar{s}_{1} \bar{s}_{1}^{+}$includes $n_{1}=4$ variables, parameter $j_{1}=3$ (equation (4)). Accordingly to equations (9) and (6), $J_{1}=0$ and thus weight of LAR $\left(y_{1}\right)$ equals $2^{J_{1}}=1$. Taking into account equation (2), $L A R\left(y_{1}\right)=2+x_{1}+x_{2}-s_{1}-s_{1}^{+}$.

The remaining LARs of the $A^{X}$ tree and their masking operators are presented in Table 1 As a result, LAR for all the cubes is equal to

$$
\begin{aligned}
A^{X} & =2^{0}\left(2+x_{1}+x_{2}-s_{1}-s_{1}^{+}\right)+2^{3}\left(4-x_{2}-s_{1}-s_{1}^{+}\right) \\
& +2^{6}\left(2-x_{1}+x_{2}-s_{1}+s_{1}^{+}\right)+2^{9}\left(2-x_{1}+s_{1}+s_{1}^{+}\right) \\
& +2^{12}\left(x_{1}+x_{2}+s_{1}+s_{1}^{+}\right)+2^{15}\left(2+x_{1}+x_{2}-s_{1}-y\right) \\
& +2^{18}\left(4-x_{2}-s_{1}-y\right)+2^{21}\left(2-x_{1}+s_{1}+y\right) \\
& +2^{24}\left(1+x_{1}+x_{2}+s_{1}-y\right) .
\end{aligned}
$$

In the first level, there are nine products $y_{t}$. The characteristic functions $\chi_{\delta}$ and $\chi_{\lambda}$ include $y_{1}$ to $y_{5}$ and $y_{6}$ to $y_{9}$, respectively. Consequently, in the second level the characteristic function $\chi_{\delta}$ can be described as $\chi_{\delta}=y_{1} \vee y_{2} \vee \ldots \vee y_{5}$ and similarly $\chi_{\lambda}$ as $\chi_{\lambda}=y_{6} \vee y_{7} \vee \ldots \vee y_{9}$.

Since $\chi_{\delta}$ includes $n_{1}=5$ literals, $j_{1}=4$ (equations (4) and (3)), the second LAR is equal to

$$
B^{Y}=2^{0}\left(7+y_{1}+y_{2}+y_{3}+y_{4}+y_{5}\right)+2^{4}\left(7+y_{6}+y_{7}+y_{8}+y_{9}\right) .
$$


Table 1. LARs and their parameters built from the cube table of the function $f$ (Example 1)

\begin{tabular}{r|rrrrr}
$t$ & \multicolumn{1}{c}{$y_{t}$} & $j_{t} J_{t}$ & \multicolumn{1}{c}{$\operatorname{LAR}\left(y_{t}\right)$} & Masking operator \\
\hline 1 & $x_{1} x_{2} \bar{s}_{1} \bar{s}_{1}^{+} 3$ & 0 & $2+x_{1}+x_{2}-s_{1}-s_{1}^{+}$ & $\boldsymbol{\Xi}_{27}^{3}\left\{A^{X}\right\}$ \\
2 & $\bar{x}_{2} \bar{s}_{1} \bar{s}_{1}^{+} 3$ & 3 & $4-x_{2}-s_{1}-s_{1}^{+}$ & $\boldsymbol{\Xi}_{27}^{6}\left\{A^{X}\right\}$ \\
3 & $\bar{x}_{1} x_{2} \bar{s}_{1} s_{1}^{+} 3$ & 6 & $2-x_{1}+x_{2}-s_{1}+s_{1}^{+}$ & $\boldsymbol{\Xi}_{27}^{9}\left\{A^{X}\right\}$ \\
4 & $\bar{x}_{1} s_{1} s_{1}^{+} 3$ & 9 & $2-x_{1}+s_{1}+s_{1}^{+}$ & $\boldsymbol{\Xi}_{27}^{12}\left\{A^{X}\right\}$ \\
5 & $x_{1} x_{2} s_{1} s_{1}^{+} 3$ & 12 & $x_{1}+x_{2}+s_{1}+s_{1}^{+}$ & $\boldsymbol{\Xi}_{27}^{15}\left\{A^{X}\right\}$ \\
6 & $x_{1} x_{2} \bar{s}_{1} \bar{y} 3$ & 15 & $2+x_{1}+x_{2}-s_{1}-y$ & $\boldsymbol{\Xi}_{27}^{18}\left\{A^{X}\right\}$ \\
7 & $\bar{x}_{2} \bar{s}_{1} \bar{y} 3$ & 18 & $4-x_{2}-s_{1}-y$ & $\boldsymbol{\Xi}_{27}^{21}\left\{A^{X}\right\}$ \\
8 & $\bar{x}_{1} s_{1} y 3$ & 21 & $2-x_{1}+s_{1}+y$ & $\boldsymbol{\Xi}_{27}^{24}\left\{A^{X}\right\}$ \\
9 & $x_{1} x_{2} s_{1} \bar{y} 3$ & 24 & $1+x_{1}+x_{2}+s_{1}-y$ & $\boldsymbol{\Xi}_{27}^{27}\left\{A^{X}\right\}$ \\
\hline
\end{tabular}

Finally, characteristics functions can be extracted with the masking operators $\chi_{\text {delta }}=$ $\Xi_{7}^{4}\left\{B^{Y}\right\}$ and $\chi_{\text {lambda }}=\boldsymbol{\Xi}_{7}^{7}\left\{B^{Y}\right\}$.

So, both of LBMDs $A^{X}$ and $B^{Y}$ representing the function $f$ include 14 non-terminal nodes and 16 terminal nodes. These LBMDs are depicted in Fig. 2]

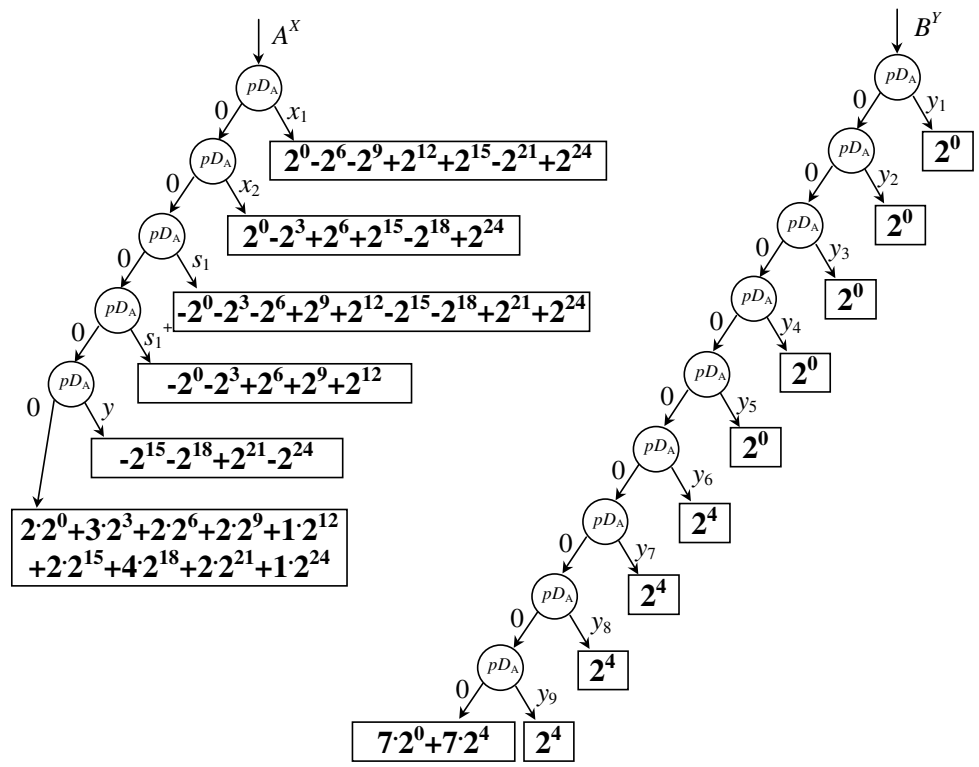

Fig. 2. Double LBMD of the function $f$ (Example 2) 


\section{Experiments}

The software implementation of the described above method were done in C++. In order to test the approach with standard benchmark library, we use FSMs given in KISS format from the LGSynth93 set. Using SIS [9], we performed a state assignment and conversion into 2-level SOP format. Each SOP, treated as the cube table of characteristic function, was then used for building a typical ROBDD using CUDD from VIS 1.3 [10] and LBMD, as proposed in this paper. Since variable ordering influences on the size of ROBDD, we used a well-known dynamic reordering method (sifting) to obtain ROBDDs of a reasonable size.

In Table 2 we give the number of nodes to represent some benchmarks by ROBDDs and LBMDs. The first two columns contain the FSM name and the number of primary inputs/outputs (I/O) and states (S). Next two columns show the number of nodes for ROBDD technique, and next columns contain the number of nodes for our LBMDs accordingly to the method described in this paper. The last two columns also contain the number of nodes for ROBBs and LBMDs, but these figures are taken from our previous work [4], where the two characteristic functions $\chi_{\delta}$ and $\chi_{\lambda}$ were united into one.

In this experiment we use JEDI from SIS to perform state assignment, which makes an assignment in natural binary code. This assignment is useful in a case of implementation into CPLD structures. From the table it follows, that the proposed approach reduces the number of nodes by 96 per cent in comparison with traditional ROBDD technique: a total number of nodes in ROBDD equals 38246, whereas in LBMDS it is equal to 1485. Moreover, a separation of two characteristic functions $\chi_{\delta}$ and $\chi_{\lambda}$ even slightly improved the result which we obtained in our previous work. However, benefits of this separation are mainly visible during the task of software simulation of a Mealy machine.

Table 2. Comparison of number of nodes of ROBDDs and LBMDs for LGSynth93 benchmarks

\begin{tabular}{|c|c|c|c|c|c|}
\hline \multirow[b]{2}{*}{ TEST } & \multirow[b]{2}{*}{ I/O/S } & \multicolumn{2}{|c|}{ Nodes in } & \multicolumn{2}{|c|}{ Nodes in $([4])$} \\
\hline & & ROBDD & LBMD & ROBDD & LBMD \\
\hline s1488 & $8 / 19 / 48$ & 4101 & 164 & 3902 & 189 \\
\hline s1494 & $8 / 19 / 48$ & 3907 & 171 & 3704 & 184 \\
\hline s208 & $11 / 2 / 18$ & 603 & 49 & 551 & 55 \\
\hline s27 & $4 / 1 / 6$ & 135 & 26 & 113 & 25 \\
\hline s298 & $3 / 6 / 218$ & 11795 & 430 & 11713 & 417 \\
\hline s386 & $7 / 7 / 13$ & 678 & 61 & 545 & 57 \\
\hline s1 & $18 / 19 / 20$ & 3321 & 142 & 3181 & 107 \\
\hline s832 & $18 / 19 / 25$ & 3031 & 128 & 2895 & 152 \\
\hline s820 & $18 / 19 / 25$ & 5008 & 138 & 5260 & 152 \\
\hline s 420 & $19 / 2 / 18$ & 640 & 55 & 587 & 61 \\
\hline s510 & $19 / 7 / 47$ & 5027 & 121 & 4855 & 104 \\
\hline Tota & & 38246 & 1485 & 37306 & 1503 \\
\hline
\end{tabular}




\section{Conclusion}

We showed that characteristic table of an arbitrary Mealy machine can be transformed to a double Linear Binary Moment Diagrams. In comparison with popular method of representing characteristic function by Reduced Ordered Decision Diagrams, we obtain diagrams with much less nodes (in our experiments we obtained a reduction equal to $96 \%)$.

Acknowledgment. The author would like to thank Prof. V.P. Shmerko and Prof. S.N. Yanushkevich for helping and making valuable comments.

\section{References}

1. R. Bryant, Y. Chen, Verification of Arithmetic Functions Using Binary Moment Diagrams, Proc. Design Automation Conf., 1995, pp. 535-541

2. J.R. Burch, E.M. Clarke, K. McMillan, Symbolic Model Checking, Fifth Annual IEEE Symposium on Logic in Computer Science, 1990, pp. 428-439

3. E.M. Clarke, E.A. Emerson, A.P. Sistla, Automatic verification of finite-state concurrent systems using temporal logic specifications, ACM Transactions on Programming Languages 8,2, 1986, pp. 244-263.

4. P. Dziurzanski , Representation of Finite State Machines with Linear Decision Diagrams, Int. Conference on Signals and Electronic Systems (ICSES'2002), Swierardow Zdroj, Poland, 2002, pp. 161-168.

5. G.D. Hachtel, F. Somenzi, Ligic Synthesis and Verification Algorithms, Kluwer Academic Publisher, 1996

6. J. E. Hopcroft, J. D. Ullman, Introduction to Automata Theory, Languages, and Computation, Addison-Wesley Publishing Company, 1979

7. V. Malyugin, Realization of Boolean Function's Corteges by Means of Linear Arithmetical Polynomial, Automation and Remote Control (USA), vol.45, no. 2, Pt. 1, 1984, pp. 239-245

8. R. Bryant, Graph - based algorithm for Boolean function manipulation, IEEE Trans. on Computers, 1986, vol. C-35, no. 8, pp. 667-691

9. SIS and Release 1.2, Logic synthesis benchmarks, UC Berkeley Soft. Distr, http:/www.cbl.ncsu.edu/www/, 1994.

10. VIS: A system for Verification and Synthesis, Proceedings of the 8th Internationa conference on Computer-Aided Verification, Springer Lecture Notes in Computer Science, \# 1102, Edited by R. Alur and T. Henziger, NJ, July 1996, pp. 428-432 